\title{
High frequency of prospecting for informed dispersal and colonisation in a social species at large spatial scale
}

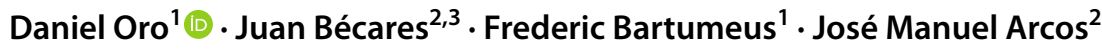

Received: 6 May 2021 / Accepted: 11 September 2021 / Published online: 22 September 2021

(C) The Author(s) 2021

\begin{abstract}
Animals explore and prospect space searching for resources and individuals may disperse, targeting suitable patches to increase fitness. Nevertheless, dispersal is costly because it implies leaving the patch where the individual has gathered information and reduced uncertainty. In social species, information gathered during the prospection process for deciding whether and where to disperse is not only personal but also public, i.e. conspecific density and breeding performance. In empty patches, public information is not available and dispersal for colonisation would be more challenging. Here we study the prospecting in a metapopulation of colonial Audouin's gulls using PTT platform terminal transmitters tagging for up to 4 years and GPS tagging during the incubation period. A large percentage of birds $(65 \%)$ prospected occupied patches; strikingly, $62 \%$ of prospectors also visited empty patches that were colonised in later years. Frequency and intensity of prospecting were higher for failed breeders, who dispersed more than successful breeders. Prospecting and dispersal also occurred mostly to neighbouring patches where population density was higher. GPSs revealed that many breeders (59\%) prospected while actively incubating, which suggests that they gathered information before knowing the fate of their reproduction. Prospecting may be enhanced in species adapted to breed in ephemeral habitats, such as Audouin's gulls. Interestingly, none of the tracked individuals colonised an empty patch despite having prospected over a period of up to three consecutive years. Lack of public information in empty patches may drive extended prospecting, long time delays in colonisation and non-linear transient phenomena in metapopulation dynamics and species range expansion.
\end{abstract}

Keywords Prospecting $\cdot$ Dispersal $\cdot$ Colonization $\cdot$ Information $\cdot$ Range expansion

\section{Introduction}

Animals explore space searching for resources such as food, shelter against predators, and mates. It is well-known that dispersal processes are common in animal populations and they have been selected to increase fitness by settling in the best patch for surviving and reproducing (Bullock et al. 2002; Clobert et al. 2012). Since patch suitability is

Communicated by Yanyun Zhang.

Daniel Oro

d.oro@csic.es

1 Centre d'Estudis Avançats de Blanes-CEAB (CSIC), Acces Cala Sant Francesc 14, 17300 Blanes, Spain

2 SEO/BirdLife-Marine Programe, Delegació de Catalunya, 08026 Barcelona, Spain

3 CORY'S-Investigación y Conservación de la Biodiversidad, 08016 Barcelona, Spain variable in space and time, prospecting is a dynamic process that allows individuals to gather information on the environmental quality of a breeding patch and make informed decisions to either stay in their current breeding patch or disperse (Reed et al. 1999). This prospecting process is also crucial for determining a species' distribution expansion range through colonisation processes and the dynamics of spatially extended metapopulations (Bowler and Benton 2005; Kokko and López-Sepulcre 2006; Clobert et al. 2009). Nevertheless, how the quality of information gathered for decision making at individual level affects metapopulation growth and dynamics remains little known (Lister 2014; Gil et al. 2018). Prospecting was first described for social birds at the scale of a single colony: individuals assessed the reproductive output of conspecifics as a clue to decide which patch of the colony offered the best chance for successful recruitment in later years (Cadiou et al. 1994; Danchin et al. 1998). Further observations were made in adult collared flycatchers shortly afterwards (Doligez et al. 1999), and a 
review highlighted that dispersal may be preceded by prospecting (i.e. the so-called informed dispersal). Some studies showed that prospecting does not always lead to dispersal (Ponchon et al. 2017) and that prospecting occurs also at larger spatial scales (Ponchon et al. 2013). Since prospecting was first noticed, there was a growing interest in assessing which were the ecological drivers influencing this behaviour. For instance, prospecting increases after perturbations (e.g. increase of predation, harsh weather) and deterioration of patch suitability (Ponchon et al. 2015a; Payo-Payo et al. 2017), but patch spatial configuration, life histories, sex, social status and social environment and its feedbacks have also been appealed (e.g. Martínez-Abraín et al. 2003; Dittmann et al. 2005; Mares et al. 2014; Hanski and Cambefort 2014; Oro 2020). Nevertheless, empirical data to assess the importance of each of the potential drivers at large spatial scales are still scarce (Votier et al. 2011; Péron and Grémillet 2013; Ponchon et al. 2015a; Campioni et al. 2017).

From an evolutionary perspective, philopatry is favoured over dispersal when environmental conditions are good and are temporally autocorrelated. Thus, information gathered in a patch is likely to be useful to reduce uncertainty and increase fitness prospects. Dispersal may be costly because it implies leaving the patch where the individual has gathered key information about the environment, such as location of both resources and threats (Bowler and Benton 2005; Bonte et al. 2012). However, the environment is not stable and variability in resource dynamics force organisms to reset decision-making about whether to stay or disperse. Theoretical models suggest that prospecting to inform dispersal decisions evolves when the costs of information gathering are low and when mortality is high during the dispersal process (Schjørring 2002; Bonte et al. 2012; Bocedi et al. 2012; Delgado et al. 2014). In social species, that information is not only personal (coming from the individual experience and performance) but can also be public at different patches. Even though the breeding performance of conspecifics has been considered the only source of public information to assess patch suitability (Danchin et al. 2004; Dall et al. 2005), several studies have found that the number of conspecifics (and heterospecifics sharing the same guild) can also be used as public information to decide where to settle (Oro and Ruxton 2001; Serrano and Tella 2003; FernándezChacón et al. 2013). This may be especially true at large spatial scales where individuals have a short lapse of time to assess patch quality using the breeding performance of conspecifics (Oro 2020). In summary, individuals of social species are likely to make dispersal decisions using private and public information (Boulinier et al. 1996; Pärt and Doligez 2003; Parejo et al. 2006; Kivelä et al. 2014). However, public information is not available in empty patches, dispersal cannot be informed and colonisation is riskier, although some benefits can be obtained compared to dispersal to an occupied patch (e.g. lack of density-dependence). Despite the importance of dispersal and its ecological and evolutionary consequences for metapopulation functioning, little is known about how often prospecting occurs, the relative frequencies of informed and non-informed dispersal. Little too is known about which drivers influence prospecting for deciding where and when to disperse including colonisation dynamics and range expansion at metapopulation scales (Delgado et al. 2014; Ponchon et al. 2015b).

Studying prospecting behaviour in nature is challenging especially for mobile species due to the large spatial scales needed for detailed individual monitoring over time. Some results have been obtained using marked individuals and monitoring their location at different breeding patches across time, but incomplete information on their reproductive status and performance, limits conclusions (e.g. Duerr et al. 2007; Henaux et al. 2007; Davis et al. 2017; Genovart et al. 2020). The most suitable method for those species is telemetry tracking to monitor individual movement and behaviour with accuracy (Votier et al. 2011; Ponchon et al. 2013, 2015a; Casazza et al. 2020). Here we study the prospecting and dispersal processes in social Audouin's gulls using a long-term monitored spatially structured population at large scale and tracked breeding individuals. The metapopulation study has historically shown an uneven clumped distribution of breeding patches, with a single patch holding up to $73 \%$ of the total world population, before experiencing a collapse due to an environmental perturbation in the form of invasive predators (Oro 2020). As a consequence, the number of occupied patches dramatically increased from 5 to 24 over the years and this allowed us to explore the colonisation process, which is difficult to track in field studies (Payo-Payo et al. 2017). Despite the small sample size, we will assess the importance of private and public information for making the decision of either stay or leave (i.e. of being philopatric or disperser). We used population size as a proxy of the available public information at each patch of the metapopulation. Since patches with larger population sizes have historically shown higher mean breeding performance (Oro et al. 1996; Cam et al. 2004; Fernández-Chacón et al. 2013; Genovart et al. 2018; Oro 2020), we could not disentangle the influence of either the breeding success of conspecifics or their densities (as different sources of public information) on dispersal decision. Larger colonies are more protected against aerial predators and they likely have more information to share about resources. Thus, we cannot disentangle which type of public information (either breeding success or the number of conspecifics, or both) was used to make the decision about either stay or disperse. However, we expected that individuals breeding in larger colonies dispersed less than those breeding in colonies with small densities, and individuals dispersing did so to larger colonies, where public information was higher. 


\section{Methods}

\section{Study species and sites}

Audouin's gulls are long-lived colonial species (mean adult survival $=0.9$ ), with a monogamous mating system and a bet-hedging life-history. Modal clutch size is three eggs, and adults incubate for ca. 28 days after the third egg is laid in late-April. Chicks are nidifugous and fledge after ca. 30 days of parental care and fledglings and adults have seasonal migration to distant wintering areas mostly in the western African coasts under the influence of the Canary current (Bécares et al. 2016).

The western Mediterranean metapopulation holds 85\% of the total world population. We have monitored population densities every year in all colonies of this metapopulation since the $80 \mathrm{~s}$ (Genovart et al. 2018) (mean number of occupied patches during 1981-2012=18; range of occupied patches: 7-26). Figure 1 shows the distribution of "occupied" breeding patches during the tracking [2006-2007 for platform terminal transmitters (PTT) and 2010-2012 for GPS tags] and "empty" patches that were colonised up to 5 years after tagging. Half of these colonisations occurred in harbours, a habitat never occupied by the species before. Breeding patch configuration changed over the years due to a perturbation occurring at the largest breeding patch at La Banya (Ebro Delta): in 1997, carnivores invaded and settled here. These carnivores (mainly foxes) have preyed on eggs, chicks and occasionally on adults, and they have also generated stress by their mere presence. It was not until several years later that many patches were colonised and La Banya experienced a rapid collapse to quasi-extinction (Payo-Payo et al. 2017; Genovart et al. 2018; Oro 2020). By colonized patches, we mean patches where gulls started to actively reproduce, building nests, mating, laying eggs and potentially raising chicks.

\section{Tracking birds}

All birds were trapped at the nest during incubation and tagged with tracking devices (Table 1). In 2006-2007, 23 birds were captured at four different colonies (Ebro Delta, Illa de l'Aire, Isla Grosa and Alboran; Fig. 1) and tagged with $18 \mathrm{~g}$ solar PTTs, using a harness, which allowed for information for up to 4 years (Bécares et al. 2016). Later in 2010-2012 another 84 birds were captured at the Ebro Delta and tagged with 25 g GPS loggers (Table 1). Sex of birds was determined by body measurement $(53 \%$ of males) but it was not included in the analysis due to the small sample size. For PTT tagging, selected data started at late incubation period of the first year (when trapped) until the device stopped emitting signals, for at least two consecutive breeding seasons. Fourteen PTT birds provided data for at least two of these seasons. Since GPS tags had to be recovered for data downloading, data corresponded only to the incubation period when birds can be trapped at the nest. PTT devices have poorer spatial resolution than GPS data and frequency was low (mean $=5$ locations per day and individual during the breeding season from April to July, range: 1-19), but 14 birds yielded at least two consecutive years of data. These data allowed us to assess the role of prospecting for breeding dispersal. GPS data were retrieved for 46 individuals, which were incubating and were actively breeding until the tag was removed by trapping the birds again. The rest of GPS were not recovered due to our inability to recapture the individual, or because they provided no information due to technical problems with the device (i.e. sealing failure, download bugs). GPS data have much higher precision and frequency (every $5 \mathrm{~min}$ ) than PTT, but tags were deployed for a maximum of 15 days.

Typically, trips performed by tracked birds during the breeding season corresponded to foraging bouts mostly at sea. For some but not all of these foraging trips, we observed gulls stopping at specific places along the coastline. In most cases, these stops occurred after a foraging bout on the way back to the colony. Out of the patches where gulls were breeding or colonised in later years, most habitats were not suitable for stopping due to intense coastal urbanisation. Two non-exclusive reasons may explain these stops out of the colony: resting and prospecting. Since tracked birds were actively breeding and constrained from returning to the colony regularly (where resting is a common behaviour), we assumed that these visits mostly corresponded to prospecting for information gathering. In other words, movements which did not occur at sea but along the coastline, most of the time after foraging on the way back to the colony were assigned as part of the prospection process, in which individuals can gather information for potential dispersal decisions. For PTT data, since devices yielded few points per day (maximum 15 points) and the spatial precision was lower (error encompassed several kilometres), locations within a radius of $7 \mathrm{~km}$ around the existing and future colonies were considered as prospecting visits. For GPS data, all stops taking longer than $10 \mathrm{~min}$ in a patch where gulls may potentially breed (other than the actual breeding patch) were considered to have been a visit for prospecting. We counted these stops as prospecting visits. In general, gulls stopped in only one patch other than their colony. For large breeding colonies, we delimitated the contours of different spatially discrete breeding patches, which were either occupied or not, in different years (Supplementary Figure S1) (Genovart et al. 2003). 

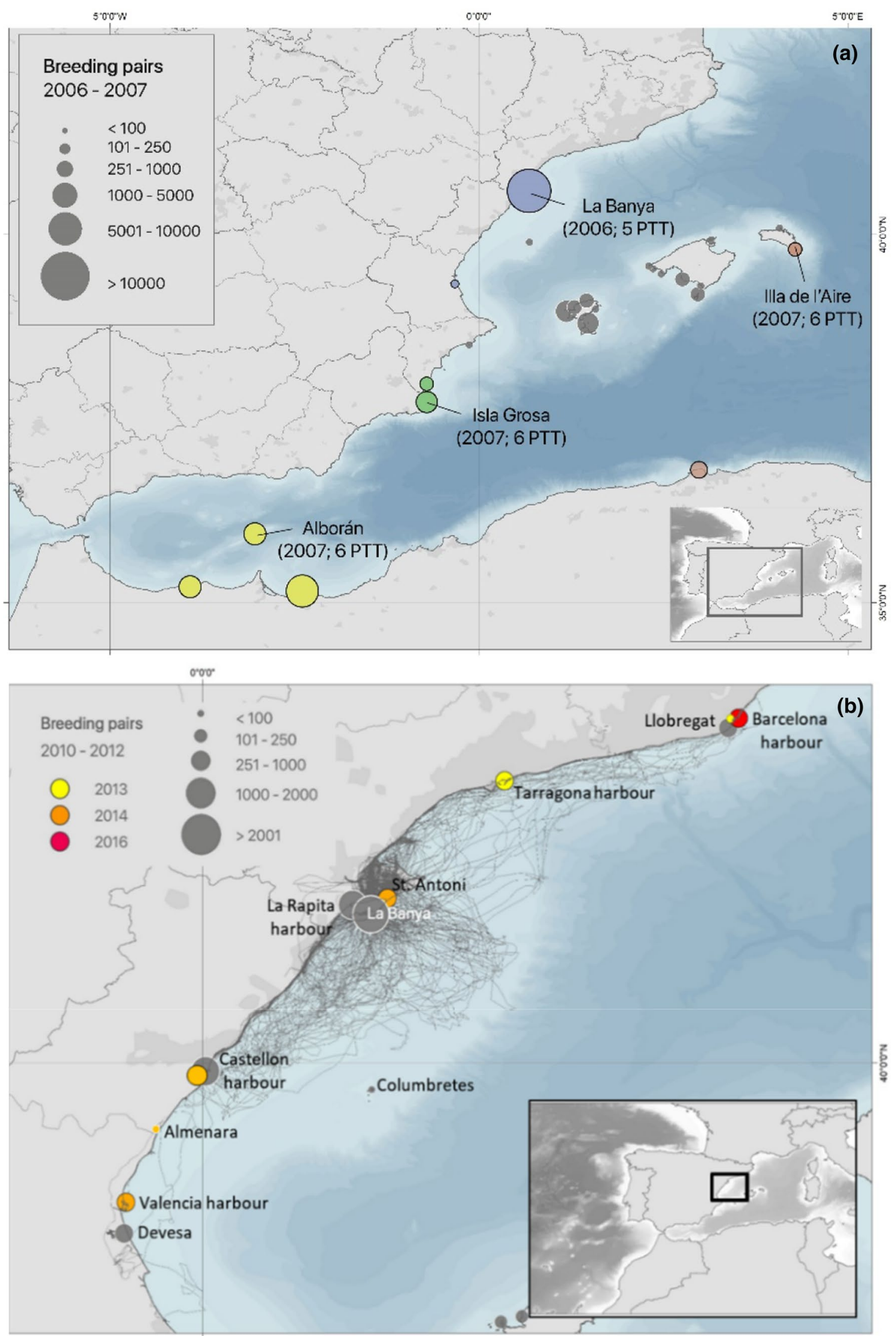
4Fig. 1 Map of the study area showing the patch spatial configuration including the study colonies where we tracked birds and the other patches (both OBP and FBP) at metapopulation scale. a Patches during PTT tracking (birds tagged in 2006-2007) showing the focal patches, the occupied patches (OBP, solid dots) and the empty patches (FBP, white dots) that were prospected during the breeding season; OBP that were not visited are shown as grey dots. Colours define sub-populations of focal patches with their prospected patches. b Patches during GPS tracking birds, all breeding at the La Banya (Ebro Delta) during 2010-2012, and trajectories for foraging and prospecting including OBP (in grey) and FBP (in different colours depending on the year of colonisation). None of the birds moved out of the area shown, during the breeding season. Size of dots for OBP show their population size during sampling

\section{Analysis of PTT tags}

The movements of each individual were analysed between April 15 and July 20 to determine whether it visited other patches and whether it failed reproduction, and how this affected whether and where it bred in the following season. Following field observations, we assumed that being absent from the colony for more than 4 days in a row away would indicate a reproductive failure (Oro 1998). We recorded the visits made by each individual to other breeding patches (Fig. 1a). Given the position error associated with the PTT system (Wilson et al. 2002), locations within a radius of $7 \mathrm{~km}$ around these colonies or future colonies (colonised up to 5 years after the tagging) were considered as visits. We assumed that a bird skipped breeding (i.e. took a sabbatical year) when it was not recorded in any colony (considering the PTT error) for more than 4 days in a row during the incubation and chick growth periods. From the 23 PTT tagged birds, 14 provided data over at least two consecutive breeding seasons (including the season when they were tagged) and allowed for the analysis of the role of breeding success and prospecting at year $t$ on breeding dispersal at year $t+1$. We considered it to be a breeding dispersal event when a gull was recorded settling in a patch during the breeding season other than the one used the previous season. We considered successful breeders (i.e. raising at least one chick) those who completed the breeding season visiting their focal breeding patch. We built GLM models to assess the factors affecting the occurrence of both dispersal and prospecting (as binomial response variables) and those affecting the intensity of prospecting measured as the number of visits to other patches (as Poisson response variable). For the occurrence of dispersal, we tested the explanatory variables of breeding failure and prospecting as categorical variables and the number of visits, and colony sizes (of both the patch of origin and the patch of destination) as continuous variable. We used the number of pairs counted at each patch as a proxy of the amount of public information available for gulls. For the occurrence of prospecting, we tested the explanatory variables of breeding failure and dispersal as categorical variables. For the intensity of prospecting, we tested the influence of breeding failure and the actual dispersal the year after on the number of visits per individual. Model selection was based on AICc values and two models with differences in AICc value $<2$ were considered statistically equivalent (Burnham and Anderson 2002).

\section{Analysis of GPS tags}

The movements of each individual were recorded with high spatio-temporal accuracy between May 13 and May 25 (corresponding to their incubation period) to determine whether the tagged individuals prospected other colonies or future breeding patches, which were mostly harbours. In total, gulls visited the following patches: Barcelona harbour, Llobregat, Tarragona harbour, La Rapita harbour, Castellon harbour and Devesa (Fig. 1b). Prospecting did not occur in colonies located within the maximum foraging range for an Audouin's gull while breeding (180 km) (see also Arcos and Oro 1996): St. Antoni, Almenara, Columbretes Is. and Valencia harbour (Fig. 1b). For the analysis, we divided these patches into two types: (1) OBP, Occupied Breeding Patches (the exact location where there were active nests while tagged birds visited the patch); and (2) FBP, future breeding patches (where colonisation occurred up to 5 years after tagging). For each individual, we recorded the number of visits to each patch type and (3) time spent by individuals visiting each patch (1-h intervals). The difference in the frequency of prospecting animals visiting OBP and FBP was tested using the Fisher's exact test. We performed an ANOVA analysis to test whether birds spent more time in OBP than in FBP. A likelihood ratio test was used to analyse whether birds visiting empty patches spent fewer nocturnal hours (when predation risk is higher) than birds visiting OBP. Finally, GLM models were built to assess the influence of distance to the Ebro Delta and population density at OBP on the occurrence of prospecting (as binomial response variable). This way, we tested the hypotheses that the occurrence of prospecting decreased with distance from the breeding patch and that the number of prospecting individuals increased with population density of the prospected patch, owing that larger colonies inform prospectors of more resources and for the study species, larger colonies have higher breeding success.

\section{Results}

Considering all tagged birds, a large percentage (65\%) prospected occupied patches, and $62 \%$ of these prospectors also visited empty patches that were colonised in later years. 
Table 1 Remote monitoring data used in our study (year of monitoring in parenthesis) at each study colony (see Fig. 1)

\begin{tabular}{llllll}
\hline Tracking device & Delta Ebro (15,329) & Aire Is (125) & Alborán Is (526) & $\begin{array}{l}\text { Grosa Is } \\
(582)\end{array}$ & Total \\
\hline PTT & $5(2006)$ & $6(2007)$ & $6(2007)$ & $6(2007)$ & 23 \\
2-years of data & 3 & 4 & 4 & 3 & 14 \\
GPS & $4(2010)$ & & & & 46 \\
& $36(2011)$ & & & \\
& $6(2012)$ & & & & \\
\hline
\end{tabular}

Colony size (as number of breeding females) is shown in parenthesis for each breeding patch. For PTT, the number of individuals monitored over two consecutive seasons is also shown

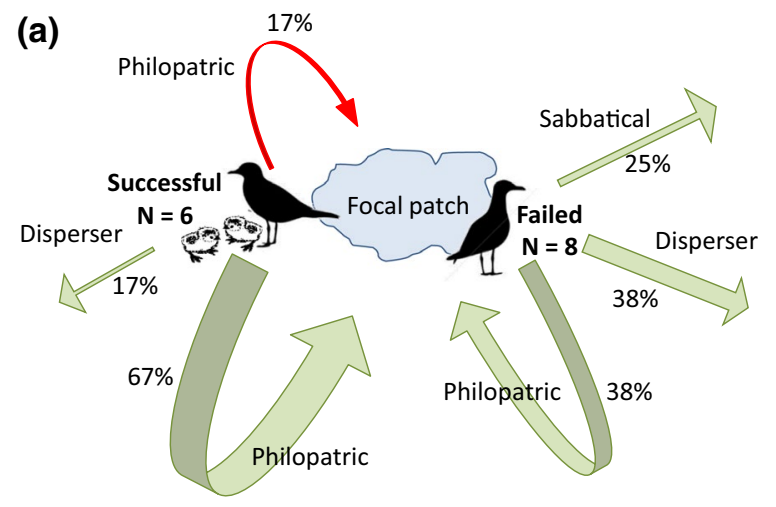

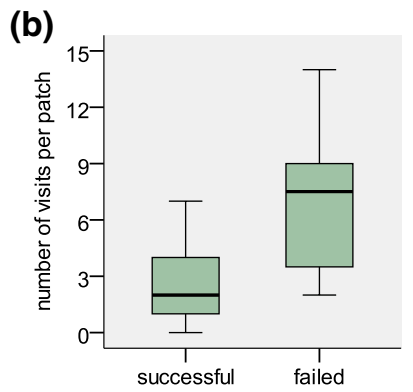

Reproduction

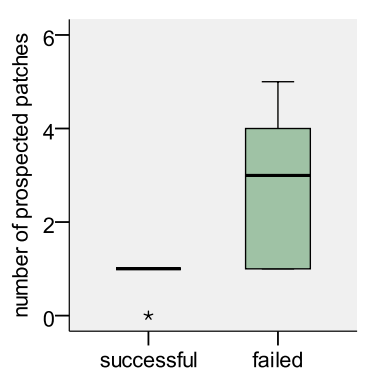

Reproduction
Fig. 2 a Graphical representation of two consecutive years of prospecting and dispersal behaviour of 14 PTT-tracked gulls tagged in 2006-2007, depending on their breeding performance. The red arrow shows the only individual that did not prospect, whereas the rest of the arrows correspond to prospecting birds. The focal patch represents the breeding patch where the birds were marked (see Fig. 1a); b Box plots of the number of visits per patch and the number of prospected patches (considering both OBP and FBP) for successful and failed breeders (color figure online)

\section{PTT tags}

Figure 2 shows the dispersal decision made by the 14 individuals monitored over two consecutive breeding seasons depending on their breeding success and their decision either to stay (philopatric, $57 \%$ of the cases), to disperse (emigrant, $29 \%$ of the cases), or to skip breeding (sabbatical, $14 \%$ of the cases). Individuals tended to be philopatric after successful reproduction, whereas failed breeders made one of the three possible decisions: being philopatric, disperse or taking a sabbatical year. Most individuals prospected at least one of the monitored seasons (93\% of total), and all failed breeders showed this behaviour. Interestingly, none of the four individuals breeding successfully and being philopatric the year after prospected other patches. The maximum distance recorded from a focal colony to an occupied breeding patch (OBP) of an active breeding individual was $164 \mathrm{~km}$. However, one failed individual performed three trips from its breeding patch to a very distant patch $360 \mathrm{~km}$ away in northern Africa, where it dispersed the following season (Fig. 1a). The small sample size precluded most GLM models to be conclusive. Selected models for dispersal included all the factors tested except the size of the colony of origin and none of them was statistically significant (Table 2). For prospecting, the best model suggests that prospecting increased for failing breeders and with the size of the prospected colony but only breeding failure was statistically significant $(\mathrm{GLM} z$ value $=2.493, P=0.013$ ). On the contrary, the intensity of prospecting was significantly higher for failed breeders than for successful breeders $($ GLM $z$ value $=2.886, P<0.004)($ Fig. $2 b)$, whereas it was not statistically different between disperser and philopatric birds $(\mathrm{GLM} z$ value $=1.454, P=0.146)$. The intensity of prospection increased with the population size of the prospected colony $($ GLM $z$ value $=5.412, P<0.001)($ Table 2$)$. The number of different patches prospected by the tracked gulls was also higher for failed than for successful breeders $\left(F_{1,13}=8.244, P=0.014\right)$ (Fig. 2b). In four cases, individuals provided data for three to four consecutive breeding seasons (Table 3). Despite the very small sample size, results are consistent with what we observed for individuals tracked over two consecutive seasons. One of these cases corresponded to a gull that skipped breeding for two consecutive years before returning to its previous breeding patch.

\section{GPS tags}

GPS tracking provided very accurate data on individuals prospecting the different patches at a large spatial scale during the breeding season (incubation period; Fig. 3). First, results show that $59 \%$ of birds prospected other patches 
Table 2 Models testing separately the factors affecting dispersal, prospecting and intensity of prospecting using data from PTT-tracked birds

\begin{tabular}{|c|c|c|c|c|c|}
\hline & $\mathrm{np}$ & Dev & $\mathrm{AICc}$ & $\Delta \mathrm{AICc}$ & Wi \\
\hline \multicolumn{6}{|l|}{ For dispersal } \\
\hline Size_dest & 2 & 15.203 & 19.203 & 0 & 0.20 \\
\hline Failing + Size_dest & 3 & 13.871 & 19.871 & 0.668 & 0.14 \\
\hline Failing & 2 & 15.992 & 19.992 & 0.789 & 0.13 \\
\hline Visits & 2 & 16.427 & 20.427 & 1.224 & 0.11 \\
\hline Size_orig + Size_dest & 3 & 15.206 & 21.206 & 2.003 & 0.07 \\
\hline Visits + Failing & 3 & 15.211 & 21.211 & 2.008 & 0.07 \\
\hline Prospect & 2 & 17.369 & 21.369 & 2.166 & 0.07 \\
\hline Failing + Size_orig & 3 & 15.578 & 21.578 & 2.375 & 0.06 \\
\hline Failing + Prospect & 3 & 15.589 & 21.589 & 2.386 & 0.06 \\
\hline Visits + Prospect & 3 & 16.344 & 22.344 & 3.141 & 0.04 \\
\hline Size_orig & 2 & 18.946 & 22.946 & 3.743 & 0.03 \\
\hline Failing + Prospect + Visits + Size_orig & 5 & 15.068 & 25.068 & 5.865 & 0.01 \\
\hline Failing + Prospect + Visits + Size_orig + Size_dest & 6 & 13.86 & 25.86 & 6.657 & 0.01 \\
\hline \multicolumn{6}{|l|}{ For prospecting } \\
\hline Failing + Size_prosp & 3 & 2.377 & 8.377 & 0.000 & 0.49 \\
\hline Failing & 2 & 5.407 & 9.4067 & 1.030 & 0.29 \\
\hline Failing + Size_orig & 3 & 5.986 & 11.986 & 3.609 & 0.08 \\
\hline Failing + Disperse & 3 & 6.142 & 12.142 & 3.765 & 0.07 \\
\hline Failing + Disperse + Size_orig + Size_prosp & 5 & 2.366 & 12.366 & 3.989 & 0.07 \\
\hline Size_prosp & 2 & 15.924 & 19.924 & 11.547 & 0.00 \\
\hline Disperse & 2 & 16.611 & 20.611 & 12.234 & 0.00 \\
\hline Size_prosp + Size_orig & 3 & 15.099 & 21.099 & 12.722 & 0.00 \\
\hline \multicolumn{6}{|l|}{ For the intensity of prospecting } \\
\hline Failing + Size_prosp & 3 & 38.734 & 44.734 & 0.000 & 0.70 \\
\hline Failing + Disperse + Size_prosp & 4 & 38.851 & 46.851 & 2.117 & 0.24 \\
\hline Size_prosp & 2 & 45.740 & 49.74 & 5.006 & 0.06 \\
\hline Failing + Disperse & 3 & 67.479 & 73.479 & 28.745 & 0.00 \\
\hline Failing & 2 & 69.704 & 73.704 & 28.97 & 0.00 \\
\hline Disperse & 2 & 77.825 & 81.825 & 37.091 & 0.00 \\
\hline
\end{tabular}

Visits number of visits, Failing whether the individual failed reproduction, Prospect whether the individual prospected, Size_origin population size at the patch of origin, Size_dest population size at the patch of destination (for dispersal), Size_prosp population size at the patch of prospection (for prospecting), $n p$ number of estimable parameters, Dev relative deviance, AICc Akaike's information criterion adjusted for small sample size $(c), \triangle A I C c$ difference between current model and the model with the lowest AICc, Wi Akaike weight of model $i$

Table 3 Reproductive status of each of the four individuals (IDs) tracked with PTT tags for more than two consecutive breeding seasons depending on whether they disperse or stay as philopatric in their colony of origin (i.e. where they were tagged)

\begin{tabular}{llllllll}
\hline ID & Colony of origin & Year $_{t}$ & $\overrightarrow{\mathrm{DA}_{t}}$ & Year $_{t+1}$ & $\overrightarrow{\mathrm{DA}}_{t+1}$ & Year $_{t+2}$ & DA $_{t+2}$ \\
\hline 33886 & Aire Is & Failure (0) & Disperse (Devesa) & Sabbatical (55) & - & Sabbatical (31) & No more signals \\
59260 & Grosa Is & Failure (21) & Disperse (Torrevieja) & Failure (1) & Philopatric & Success (3) & Philopatric \\
33888 & Alborán Is & Success (1) & Philopatric & Failure (0) & Philopatric & Failure (4) & Philopatric \\
65796 & Alborán Is & Success (0) & Philopatric & Success (5) & Philopatric & Failure (36) & No more signals \\
\hline
\end{tabular}

Numbers in parenthesis are the number of days prospecting other patches during the breeding season. $D A$ decision after the breeding season in year $t$. Data for the two first years $t$ and $\mathrm{t}+1$ are already included in Fig. 2 and Table 2. When an individual dispersed, the colony of destination is also shown in parenthesis 


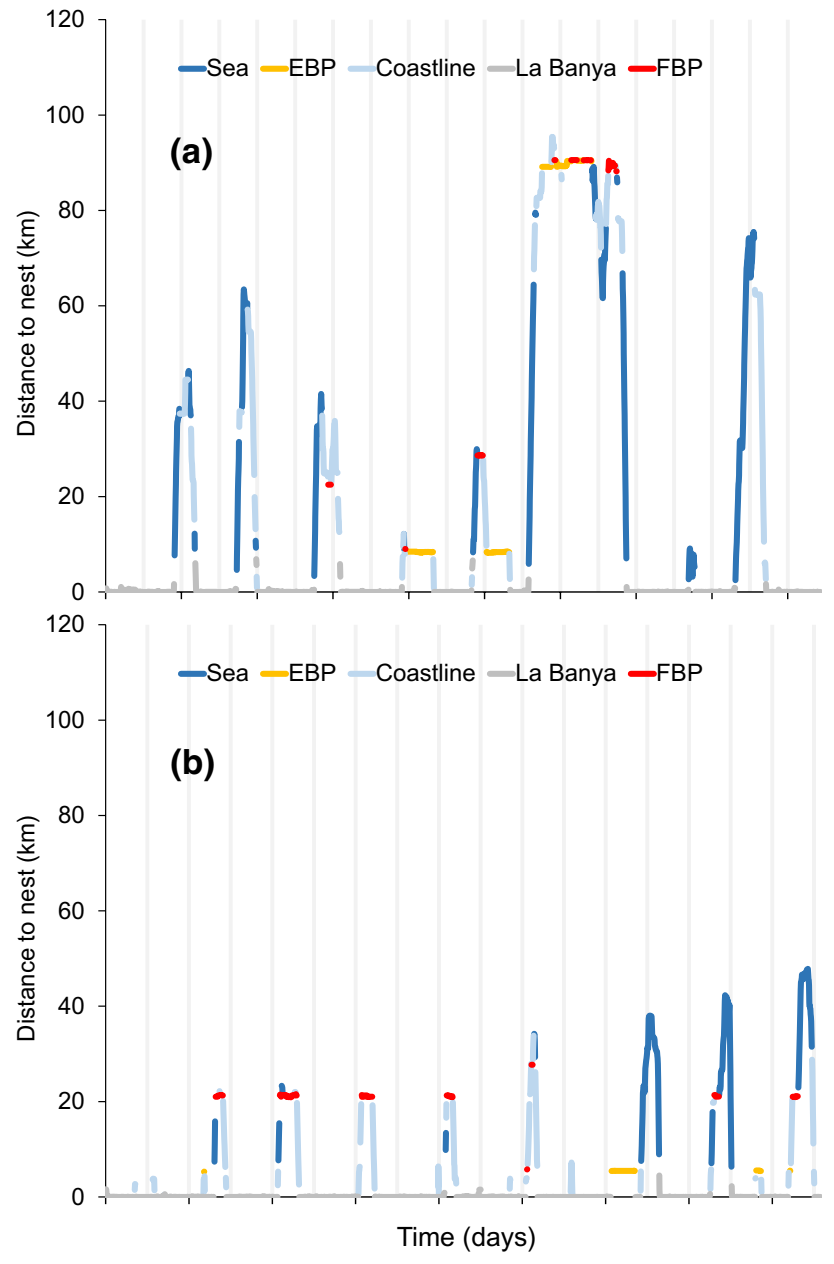

Fig. 3 Two examples of GPS tracking data for individuals 5,107,916 and 5,107,927 (upper and lower panel $\mathbf{a}$ and $\mathbf{b}$ respectively) while breeding at La Banya. The graph shows the movements of these individuals over 9.5 consecutive days (vertical lines shows $12 \mathrm{~h}$-intervals) corresponding to the late incubation period just before chick hatching. Consecutive points forming a horizontal line indicate no movement, which corresponded to stays either at the nest [for values of $Y$ (distance to the nest) $=0$ ], or prospecting (sometimes lasting up to $10 \mathrm{~h}$ ) at other patches (OBP and FBP) (see Fig. 1b). Movements included several trips out of the colony, many of them to prospect

before potential reproductive failure (i.e. while actively breeding): $50 \%$ prospected only OBP, $25 \%$ prospected only FBP (i.e. empty patches) that were colonised $1-5$ years later, and $25 \%$ of birds prospected both occupied and empty patches (Table 4 ). The percentage of prospecting animals visiting OBP was higher than that visiting FBP, but the difference was not statistically significant (Fisher's exact test, $P=0.290$ ). The number of prospecting gulls per patch (including OBP and FBP) decreased with the distance to the focal colony $(\mathrm{GLM} z$ value $=-2.444, P<0.02)($ Table 4$)$. The number of prospecting gulls in OBP increased with population density at the patch $(\mathrm{GLM} z$ value $=3.401, P<0.0001)$ (Table 4, Fig. 1b). Birds on average spent more time in OBP with breeding conspecifics than in FBP that were colonised in later years $\left(F_{1,68}=5.539, P=0.021\right)$. Finally, birds prospected OBP and FBP at different times of the day: for OBP, the frequency of individuals prospecting at each daytime followed the same pattern of fluctuations in the number of birds present at colonies (Oro 1995, 1998), peaking at night, whereas birds visiting empty patches prospected at nocturnal hours significantly less (Likelihood Ratio test $=547.7, d f=1$, $P<0.0001)$ (Fig. 4).

\section{Discussion}

Results from PTT-tracked Audouin's gulls provide some insight on patterns on prospecting and breeding dispersal, although sample size was relatively small. First, breeding dispersal was much higher for failed breeders than for birds successfully breeding. Breeding failure is a very common driver for dispersal (Ronce 2007; Matthysen 2012), and personal information on individual own breeding performance is used to decide either to stay or disperse (Parejo et al. 2006). However, our results also suggest that this decision was fully made only after prospecting. All failed breeders prospected, and dispersal was more frequent for individuals prospecting more patches and performing more visits to these patches. This confirms the crucial role of prospecting to inform future dispersal events (Reed et al. 1999; Clobert et al. 2009). It is thus not surprising that prospecting is a widespread behaviour used by an individual to decide first if there is enough information to leave its patch and second, in which patch it would settle (Stamps 1988; Reed et al. 1999; Dall et al. 2005; Clobert et al. 2009; Schmidt et al. 2010; Morales et al. 2010). Prospecting would have been selected over evolutionary scales due to the potential benefits for fitness prospects, e.g. by lowering mortality (Kingma et al. 2016). These benefits result from gathering information (both personal and social) at alternative patches, which would reduce the potential risks of dispersal (Schmidt et al. 2010; Delgado et al. 2014; Ponchon et al. 2015b). Interestingly, we found that failed Audouin's gulls gathering more information (i.e. prospecting several patches both occupied and empty) had more chances to disperse than those prospecting a single patch. At the scale of a single colony structured in spatially discrete sub-colonies, some previous studies on other social seabirds also show that individuals recruited at the most prospected sub-colony over the previous season (Dittmann et al. 2007). Further studies are needed to determine more precisely how the environment shapes prospecting in addition to all the other factors (e.g. occurrence of sociality, sex, personality, life-history strategies). Our results would apply for species occupying ephemeral habitats (e.g. coprophagous insects, species benefiting from fires and from unpredictable pulses of resources) and 


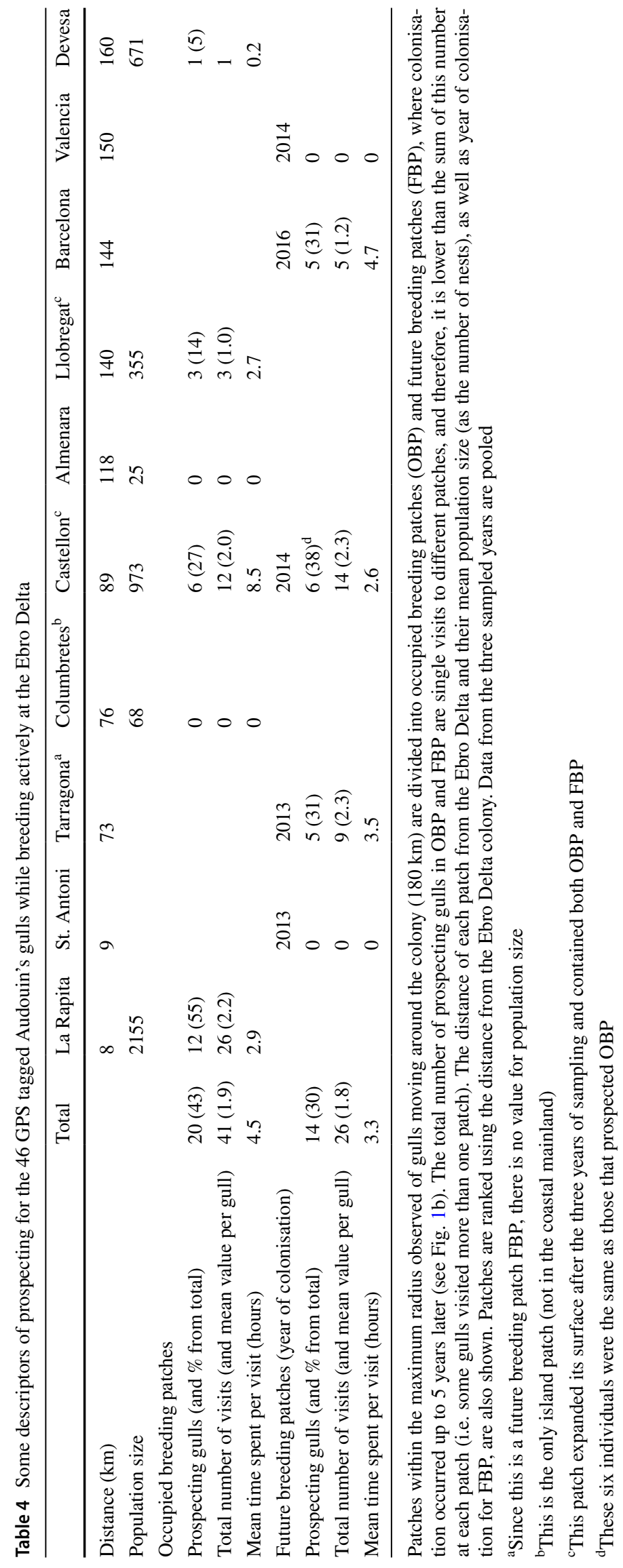




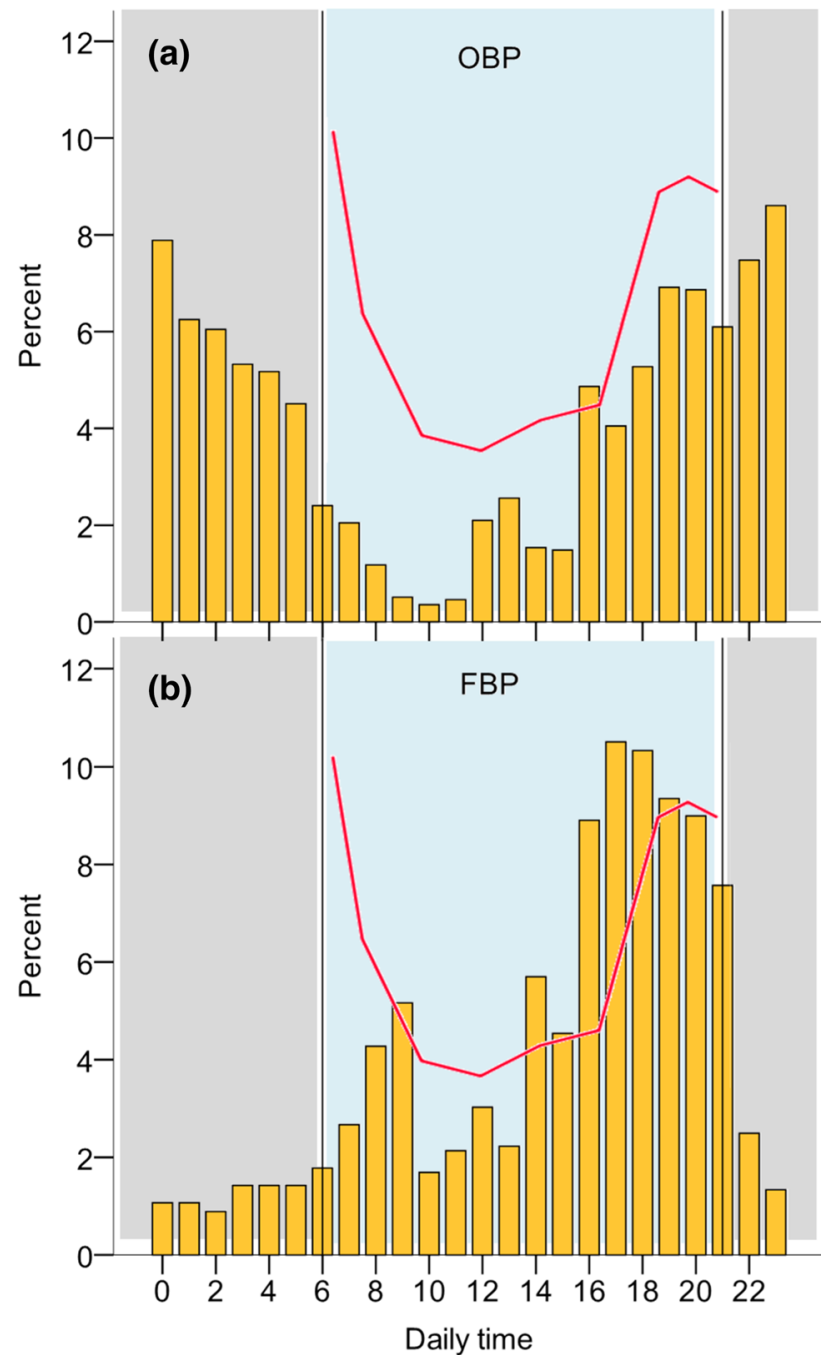

Fig. 4 Percentage of GPS locations for birds breeding at La Banya (yellow bars) and prospecting established breeding patches (OBP, upper panel a) and patches that were colonised in later years (FBP, lower panel b) in the same colony (Castellon harbour, Fig. 1) depending on daily time. Red line shows the relative density of breeders at daylight time in an occupied colony (adapted from Oro 1995) and is shown as a reference of the variation in density over that time compared to prospecting birds. The difference between patches was the presence of conspecifics in OBP (i.e. public information available). Data for the three sampled years are pooled

habitats with high spatio-temporal variability due to the stochasticity of physical drivers (e.g. marshlands, dunes, seasonal streams).

Even though we only analysed prospecting for breeding dispersal, previous studies on other species show that prospecting is also important for recruitment, i.e. the patch where individuals decide to incorporate to the breeding part of the population. Once again, recruiters may be philopatric (i.e. return to their natal colony to breed) or instead prospect other patches using public information (i.e. breeding success and the presence of both conspecifics and heterospecifics) to make natal dispersal decisions (Schjørring 2002; Ward 2005; Dittmann et al. 2007; Bosman et al. 2013; Casazza et al. 2020). For Audouin's gulls, recruitment is mostly informed, meaning that it occurs more often when the individual has prospected the patch the year before (Genovart et al. 2020). Individuals recruiting without prospecting were all young first-time breeders affected by demographic carryover effects, i.e. they were more reluctant to reproduce in subsequent seasons than individuals that recruited after prospecting. Prospecting in non-breeding birds has particular features compared to that performed by breeders. The former does not have energetic and spatial constraints imposed by reproduction, they have limited personal information (they have never bred before), and it seems that they mostly prospect late in the breeding season when public information on breeding success of conspecifics in different patches is available (Boulinier et al. 1996; Ward 2005; Greenville et al. 2016; Sherer 2019; Brandl et al. 2019; Tolvanen et al. 2020).

Our GPS results, using a larger sample size and higher spatial resolution, confirmed that prospecting in breeding Audouin's gulls was a very common behaviour. Strikingly, this prospecting occurred while Audouin's gulls were actively incubating, as it was found for Sandwich terns Thalasseus sandvicensis (Fijn et al. 2014). Contrarily, previous studies on social kittiwakes Rissa tridactyla found that prospecting among breeders only occurred after failing reproduction (Ponchon et al. 2015a, 2017). On the other hand, the occurrence of prospecting in Audouin's gulls, as it was previously recorded for breeding dispersal, decreased with the distance from the focal patch (Oro and Pradel 1999; Fernández-Chacón et al. 2013; Bécares et al. 2016). At small distances, animals may revisit prospected patches to gather more information, to update information and to enhance informed dispersal, whereas distant patches require more sequential searches (Selonen and Hanski 2010). Furthermore, prospecting occurred more often in patches holding larger population densities, likely because they yielded higher public information and they indicate a large availability of resources (Cam et al. 2004; Fernández-Chacón et al. 2013; Payo-Payo et al. 2017; Genovart et al. 2020). Furthermore, results of Cam et al. (2004) on the study species did not support the hypotheses that dispersal was influenced by mean breeding success in the colony of origin (i.e. an indicator of habitat quality) or in the destination colony, or by the ratio of breeding success in these colonies (i.e. quality gradient). It seems that private information and colony size are the main clues used by Audouin's gulls to make the decision about stay or leave the patch (Parejo et al. 2006; Fernández-Chacón et al. 2013). Studies dealing with other social birds also found that patches with higher population densities attract more individuals (Serrano and Tella 2003; Dittmann et al. 2005; Péron et al. 2010). Public information in the form of the number of conspecific at each patch is 
particularly conspicuous for social species since population density is a clue to readily assess patch suitability at large spatial scales (Nocera et al. 2006; Evans et al. 2016; Oro 2020). Some theoretical models highlight the role of densitydependence in breeding patch selection and of spatial heterogeneity in patch suitability (Nurmi et al. 2017).

\section{Drivers of prospecting}

It was clear that Audouin's gull changed their prospecting behaviour in empty patches compared to breeding patches where conspecifics were present. In occupied patches, prospecting visits overlapped with the numbers of breeders present, which suggest that prospectors look for higher density of conspecifics, either to increase information gathering or to increase protection against predation. On the contrary, prospecting birds avoided nocturnal time in empty patches, when the number of conspecifics to warn about the presence of predators is lower. Previous studies on the same species show that dispersal to occupied patches is performed mostly by younger breeders after a short, pulsed perturbation (Oro et al. 1999). Nevertheless, press perturbations (i.e. those that last several breeding seasons) may trigger colonizations of empty patches, and dispersal to these empty patches is performed by older, more experienced breeders (PayoPayo et al. 2017, 2018). Despite our small sample size, our results also suggest that birds tend to disperse to patches where population density is larger, since these patches show higher breeding success. As more studies assess the occurrence of prospecting, it becomes clearer that it is a universal behaviour selected to reduce the costs of dispersal. Can our results on Audouin's gulls be extrapolated to other species? Prospecting seems to be a sine-qua-non condition for dispersal, and drivers influencing the two processes would show a common pattern across taxa, but also specificities. First, the frequency of prospecting would decrease with patch stability (Schjørring 2002), since stability increases the temporal autocorrelation in patch quality (Danchin et al. 1998; Oro 2020). For instance, Procellariiformes are seabirds that breed in very stable habitats, and prospecting occurs mostly before recruitment, whereas once recruited, birds seldom prospect even after repeated breeding failures (Igual et al. 2007; Jenouvrier et al. 2008; Campioni et al. 2017). On the other extreme, species breeding in ephemeral habitats, such as Audouin's gulls, terns and flamingos, would show the highest occurrence of prospecting (Erwin et al. 1998; Oro 2002; Oro et al. 2009; Acker et al. 2018; Francesiaz et al. 2020). Besides, the spatial configuration of patches, such as distance between patches and heterogeneity in quality, influences the occurrence of prospecting, which may be constrained by habitat loss and fragmentation (Schmidt 2017). The capacity of different species for prospecting at large spatial scales, especially in vagile species, may be higher than previously detected due to technological challenges and research biases (Cooper and Marra 2020). We found that one Audouin's gull, after failing reproduction, was able to prospect at a very distant patch $(360 \mathrm{~km})$ where it dispersed the following season. We have mentioned that failing reproduction (or poor breeding performance) stimulates prospecting across taxa (Duerr et al. 2007; Ponchon et al. 2015a; Payo-Payo et al. 2017; Spendelow and Eichenwald 2018). Since breeding performance greatly depends on environmental stochastic conditions, harsher environment (e.g. habitat density-dependence, perturbations) would increase prospecting (Rémy et al. 2011; Payo-Payo et al. 2018; Sherer 2019). Nevertheless, prospecting may be increased in years of better environmental conditions, when physiological stress and costs of prospecting may be lower, as it has been recorded for social meerkats Suricata suricatta (Mares et al. 2014).

Besides environmental features, prospecting also varies within populations, depending on personalities (Schuett et al. 2012; Burkhalter et al. 2015), age, and sex (Doligez et al. 2004; Ward 2005; Wolfson et al. 2020), but data are still scarce to define a general pattern of their influence. Little is also known about the role of life histories, because even though most data come from long-lived species, shortlived species may also prospect (Pärt et al. 2011). Finally, social species have specific drivers of prospecting, such as social status and social environments (Mares et al. 2014; Kingma et al. 2016). In these species, recent studies show the importance of the social context of animals moving in groups and how decisions about where to go, to settle and to gather information would depend on what others decide (Mares et al. 2014; Strandburg-Peshkin et al. 2018; Oro 2020).

\section{Influence of prospecting on population and metapopulation dynamics}

Since dispersal is mostly preceded by prospecting, prospecting may influence the local population and metapopulation dynamics (e.g. extinction-colonisation rates) (Delgado et al. 2011). Prospecting and informed dispersal also create asymmetric movements from degrading patches towards better patches, generating a heterogeneous distribution of individuals into the different patches (Ponchon et al. 2015b). In social species, many processes, such as information gathering and social copying, are density-dependent and generate feedbacks resulting in non-linear extinctions and population growth (Oro and Ruxton 2001; Gil et al. 2018). Individual decisions in social species partly depend on what others decide, and prospecting before leaving a patch and colonisation are processes that may generate non-linear transient phenomena in metapopulation dynamics (Hastings et al. 2018). In our study, none of the PTT-tracked individuals colonised a patch even though some gulls prospected empty 
patches for at least three consecutive years. Prospecting may last several years before deciding to settle after group dispersal (Morales et al. 2010; Munilla et al. 2016; Payo-Payo et al. 2017; Robinson et al. 2019; Oro 2020). Audouin's gulls spent less time in empty breeding patches such as harbours, a novel habitat that was colonised in later years, and individuals gathered enough information for informed dispersal only after repeated visits over the years. Prospecting can be especially long when individuals explore novel environments where there is no public information about their suitability and this may lower colonisation rates that would ensure metapopulation performance and species range expansion (Schippers et al. 2009; Delgado et al. 2011; Schuett et al. 2012).

\section{Conclusions}

It is assumed that animals move between patches to attain resources (e.g. food, shelter, and mates) for increasing fitness prospects. The other side of the coin is that stops between movement batches would correspond mostly to resting. Nevertheless, environmental stochasticity at spatiotemporal scales affects the amount of available resources and prospecting is a necessary process to gather and update information about the suitability of a patch. At ecological level, prospecting can also be useful when the environment is heterogeneous and is temporally auto-correlated (Danchin et al. 2004). Prospecting is a dynamic process requiring both movements for exploring space and stops for gathering information in a patch. Although prospecting at large scales has been explored for a limited range of species, it should increase for species evolving in very dynamic patches such as ephemeral habitats. Audouin's gulls are social seabirds breeding in beaches and marshes that may change their patch availability and suitability from year to year. Our work shows that gulls prospect at large spatial scales while they are breeding, looking for suitable patches to disperse in case of conditions at the present patch would deteriorate. Although failing breeding promotes prospecting to increase fitness through dispersal, gulls having success also prospected, which confirms that gathering information is a key mechanism allowing organisms to make informed dispersal decisions (Clobert et al. 2009; Ponchon et al. 2015b). We show that stops at occupied patches were shorter than stops at empty patches, where public information is lack. In empty patches, information is gathered at a multi-annual scale and colonization of these patches would occur only after ensuring their suitability for breeding. Furthermore, an increase in prospecting may be a warning signal of deteriorating environmental conditions at the actual breeding patch. Since prospecting affects the ability to disperse by reducing the risks of leaving the actual patch, prospecting may affect metapopulation dynamics and the extinction-colonization turnover.

Supplementary Information The online version contains supplementary material available at https://doi.org/10.1007/s00442-021-05040-4.

Acknowledgements Victor García-Matarranz (MITECO, Spanish Government) was of great help with the tagging of birds, while many other people assisted in the field, including Raül Escandell, David García, Manuel García-Tarrasón, Gonzalo González-Barberá, Lluís Jover, Juan Carlos Nevado, Àngels Ribas, Beneharo Rodríguez, Ángel Sallent, Carola Sanpera, José Torrent and Carlota Viada. We thank Meritxell Genovart, Aurore Ponchon and an anonymous reviewer for suggestions that greatly improved the manuscript.

Author contribution statement DO conceived of the study and led writing; FB supported writing and analysis; JB and JMA lead fieldwork, data collection and analysis and supported writing. All authors gave final approval for publication and agree to be held accountable for the work performed therein.

Funding Open Access funding provided thanks to the CRUE-CSIC agreement with Springer Nature. This work was supported by Projects LIFE "Important bird areas for seabirds (Marine IBAs) in Spain" (2004-2009; LIFE04NAT/ES/000049) and LIFE + INDEMARES (2009-2014; LIFE07NAT/E/000732), and was related to actions conducted by SEO/BirdLife. Funds were partially provided by the Spanish Ministry of Science (Grant ref. CGL2017-85210-P).

Availability of data and materials The dataset supporting conclusion in this article is available at the Seabird Tracking Database of BirdLife International (http://www.seabirdtracking.org/).

\section{Declarations}

Conflict of interest The authors declare that they have no competing interests.

Ethics approval and consent to participate Trapping and tagging of Audouin's gulls was conducted with the necessary permits from the regional governments of Catalunya, Balears, Murcia and Andalucía, as well as from the Ebro Delta Natural Park.

Consent for publication Not applicable.

Open Access This article is licensed under a Creative Commons Attribution 4.0 International License, which permits use, sharing, adaptation, distribution and reproduction in any medium or format, as long as you give appropriate credit to the original author(s) and the source, provide a link to the Creative Commons licence, and indicate if changes were made. The images or other third party material in this article are included in the article's Creative Commons licence, unless indicated otherwise in a credit line to the material. If material is not included in the article's Creative Commons licence and your intended use is not permitted by statutory regulation or exceeds the permitted use, you will need to obtain permission directly from the copyright holder. To view a copy of this licence, visit http://creativecommons.org/licenses/by/4.0/. 


\section{References}

Acker P, Francesiaz C, Béchet A et al (2018) Insights on dispersal and recruitment paradigms: sex- and age-dependent variations in a nomadic breeder. Oecologia 186:85-97. https://doi.org/10. 1007/s00442-017-3972-7

Arcos JM, Oro D (1996) Changes in foraging range of Audouin's Gull Larus audouinii in relation to a trawler moratorium in the western Mediterranean. Col Waterbirds 19:128-131

Bécares J, Arcos JM, Oro D (2016) Migration and spatial ecology of the Audouin's gull in the western Mediterranean and Northwest Africa. SEO/BirdLife, Madrid, Spain

Bocedi G, Heinonen J, Travis JMJ (2012) Uncertainty and the role of information acquisition in the evolution of context-dependent emigration. Am Nat 179:606-620. https://doi.org/10.1086/ 665004

Bonte D, Dyck HV, Bullock JM et al (2012) Costs of dispersal. Biol Rev 87:290-312. https://doi.org/10.1111/j.1469-185X.2011. 00201.x

Bosman DS, Vercruijsse HJP, Stienen EWM et al (2013) Age of first breeding interacts with pre- and post-recruitment experience in shaping breeding phenology in a long-lived gull. PLoS ONE 8:e82093. https://doi.org/10.1371/journal.pone.0082093

Boulinier T, Danchin E, Monnat JY et al (1996) Timing of prospecting and the value of information in a colonial breeding bird. J Avian Biol 27:252-256

Bowler DE, Benton TG (2005) Causes and consequences of animal dispersal strategies: relating individual behaviour to spatial dynamics. Biol Rev 80:205-225. https://doi.org/10.1017/S146479310 4006645

Brandl HB, Griffith SC, Laaksonen T, Schuett W (2019) Begging calls provide social cues for prospecting conspecifics in the wild Zebra Finch (Taeniopygia guttata). Auk. https://doi.org/10.1093/ auk/ukz007

Bullock JM, Kenward RE, Hails RS (2002) Dispersal ecology. Cambridge University Press, Cambridge

Burkhalter JC, Fefferman NH, Lockwood JL (2015) The impact of personality on the success of prospecting behavior in changing landscapes. Curr Zool 61:557-568. https://doi.org/10.1093/ czoolo/61.3.557

Burnham KP, Anderson DR (2002) Model selection and multimodel inference: a practical information-theoretic approach. Springer, New York

Cadiou B, Monnat JY, Danchin E (1994) Prospecting in the kittiwake, Rissa tridactyla: different behavioural patterns and the role of squatting in recruitment. Anim Behav 47:847-856. https://doi. org/10.1006/anbe.1994.1116

Cam E, Oro D, Pradel R, Jimenez J (2004) Assessment of hypotheses about dispersal in a long-lived seabird using multistate capturerecapture models. J Anim Ecol 73:723-736

Campioni L, Granadeiro JP, Catry P (2017) Albatrosses prospect before choosing a home: intrinsic and extrinsic sources of variability in visit rates. Anim Behav 128:85-93. https://doi.org/10.1016/j. anbehav.2017.04.008

Casazza ML, McDuie F, Lorenz AA et al (2020) Good prospects: high-resolution telemetry data suggests novel brood site selection behaviour in waterfowl. Anim Behav 164:163-172. https:// doi.org/10.1016/j.anbehav.2020.04.013

Clobert J, Galliard J-FL, Cote J et al (2009) Informed dispersal, heterogeneity in animal dispersal syndromes and the dynamics of spatially structured populations. Ecol Lett 12:197-209. https:// doi.org/10.1111/j.1461-0248.2008.01267.x

Clobert J, Baguette M, Benton TG et al (2012) Dispersal ecology and evolution. Oxford University Press, Oxford
Cooper NW, Marra PP (2020) Hidden long-distance movements by a migratory bird. Curr Biol 30:4056-4062.e3. https://doi.org/10. 1016/j.cub.2020.07.056

Dall SRX, Giraldeau L-A, Olsson O et al (2005) Information and its use by animals in evolutionary ecology. Trends Ecol Evol 20:187193. https://doi.org/10.1016/j.tree.2005.01.010

Danchin E, Boulinier T, Massot M (1998) Conspecific reproductive success and breeding habitat selection: implications for the study of coloniality. Ecology 79:2415-2428

Danchin É, Giraldeau L-A, Valone TJ, Wagner RH (2004) Public information: from nosy neighbors to cultural evolution. Science 305:487-491. https://doi.org/10.1126/science.1098254

Davis KL, Schoenemann KL, Catlin DH et al (2017) Hatch-year Piping Plover (Charadrius melodus) prospecting and habitat quality influence second-year nest site selection. Auk 134:92-103. https:// doi.org/10.1642/AUK-16-80.1

Delgado MM, Bartoń KA, Bonte D, Travis JMJ (2014) Prospecting and dispersal: their eco-evolutionary dynamics and implications for population patterns. Proc R Soc B 281:20132851. https://doi. org/10.1098/rspb.2013.2851

DelgadoRatikainenKokko MMIIH (2011) Inertia: the discrepancy between individual and common good in dispersal and prospecting behaviour. Biol Rev 86:717-732. https://doi.org/10.1111/j. 1469-185X.2010.00167.x

Dittmann T, Zinsmeister D, Becker PH (2005) Dispersal decisions: common terns, Sterna hirundo, choose between colonies during prospecting. Anim Behav 70:13-20

Dittmann T, Ezard THG, Becker PH (2007) Prospectors' colony attendance is sex-specific and increases future recruitment chances in a seabird. Behav Processes 76:198-205

Doligez B, Danchin E, Clobert J, Gustafsson L (1999) The use of conspecific reproductive success for breeding habitat selection in a non-colonial, hole-nesting species, the collared flycatcher. J Anim Ecol 68:1193-1206. https://doi.org/10.1046/j.1365-2656. 1999.00362.x

Doligez B, Pärt T, Danchin E (2004) Prospecting in the collared flycatcher: gathering public information for future breeding habitat selection? Anim Behav 67:457-466. https://doi.org/10.1016/j. anbehav.2003.03.010

Duerr AE, Donovan TM, Capen DE (2007) Management-induced reproductive failure and breeding dispersal in double-crested cormorants on Lake Champlain. J Wildl Manag 71:2565-2574. https://doi.org/10.2193/2006-527

Erwin RM, Nichols JD, Eyler TB et al (1998) Modeling colony-site dynamics: a case study of Gull-billed terns (Sterna nilotica) in coastal Virginia. Auk 115:970-978

Evans JC, Votier SC, Dall SRX (2016) Information use in colonial living. Biol Rev 91:658-672. https://doi.org/10.1111/brv.12188

Fernández-Chacón A, Genovart M, Pradel R et al (2013) When to stay, when to disperse and where to go: survival and dispersal patterns in a spatially structured seabird population. Ecography 36:1117-1126. https://doi.org/10.1111/j.1600-0587.2013.00246.x

Fijn RC, Wolf P, Courtens W et al (2014) Post-breeding prospecting trips of adult Sandwich Terns Thalasseus sandvicensis. Bird Study 61:566-571. https://doi.org/10.1080/00063657.2014.950942

Francesiaz C, Yohannes E, Besnard A et al (2020) Foraging niche shift maintains breeding parameters of a colonial waterbird during range expansion. Ecol Evol 10:1988-1997. https://doi.org/ 10.1002/ece 3.6030

Genovart M, Jover L, Ruiz X, Oro D (2003) Offspring sex ratios in subcolonies of Audouin's gull, Larus audouinii, with differential breeding performance. Can J Zool 81:905-910

Genovart M, Oro D, Tenan S (2018) Immature survival, fertility, and density dependence drive global population dynamics in a longlived species. Ecology 99:2823-2832. https://doi.org/10.1002/ ecy. 2515 
Genovart M, Oro D, Choquet R, Pradel R (2020) Informed recruitment or the importance of taking stock. bioRxiv. arXiv:2005.06248

Gil MA, Hein AM, Spiegel O et al (2018) Social information links individual behavior to population and community dynamics. Trends Ecol Evol 33:535-548. https://doi.org/10.1016/j.tree.2018.04.010

Greenville AC, Wardle GM, Nguyen V, Dickman CR (2016) Spatial and temporal synchrony in reptile population dynamics in variable environments. Oecologia 182:475-485. https://doi.org/10.1007/ s00442-016-3672-8

Hanski I, Cambefort Y (2014) Dung beetle ecology. Princeton University Press, Princeton

Hastings A, Abbott KC, Cuddington K et al (2018) Transient phenomena in ecology. Science 361:eaat6412. https://doi.org/10.1126/ science.aat6412

Henaux V, Bregnballe T, Lebreton J-D (2007) Dispersal and recruitment during population growth in a colonial bird, the great cormorant Phalacrocorax carbo sinensis. J Avian Biol 38:44-57. https://doi.org/10.1111/j.2006.0908-8857.03712.x

Igual JM, Forero MG, Gomez T, Oro D (2007) Can an introduced predator trigger an evolutionary trap in a colonial seabird? Biol Cons 137:189-196. https://doi.org/10.1016/j.biocon.2007.02. 003

Jenouvrier S, Tavecchia G, Thibault J-C et al (2008) Recruitment processes in long-lived species with delayed maturity: estimating key demographic parameters. Oikos 117:620-628. https://doi.org/10. 1111/j.0030-1299.2008.16394.x

Kingma SA, Bebbington K, Hammers M et al (2016) Delayed dispersal and the costs and benefits of different routes to independent breeding in a cooperatively breeding bird. Evolution 70:2595-2610. https://doi.org/10.1111/evo.13071

Kivelä SM, Seppänen J-T, Ovaskainen O et al (2014) The past and the present in decision-making: the use of conspecific and heterospecific cues in nest site selection. Ecology 95:3428-3439. https:// doi.org/10.1890/13-2103.1

Kokko H, López-Sepulcre A (2006) From individual dispersal to species ranges: perspectives for a changing world. Science 313:789_ 791. https://doi.org/10.1126/science.1128566

Lister BC (2014) Information, behaviour and population dynamics. Oikos 123:1431-1438. https://doi.org/10.1111/oik.01423

Mares R, Bateman AW, English S et al (2014) Timing of predispersal prospecting is influenced by environmental, social and statedependent factors in meerkats. Anim Behav 88:185-193. https:// doi.org/10.1016/j.anbehav.2013.11.025

Martínez-Abraín A, Oro D, Forero MG, Conesa D (2003) Modeling temporal and spatial colony-site dynamics in a longlived seabird. Popul Ecol 45:133-139. https://doi.org/10.1007/ s10144-003-0150-Z

Matthysen E (2012) Multicausality of dispersal: a review. In: Clobert J, Baguette M, Benton TG, Bullock JM (eds) Dispersal ecology and evolution. Oxford University Press, Oxford, pp 3-18

Morales JM, Moorcroft PR, Matthiopoulos J et al (2010) Building the bridge between animal movement and population dynamics. Philos Trans R Soc B 365:2289-2301. https://doi.org/10.1098/ rstb.2010.0082

Munilla I, Genovart M, Paiva VH, Velando A (2016) Colony foundation in an oceanic seabird. PLoS ONE 11:e0147222. https://doi. org/10.1371/journal.pone.0147222

Nocera JJ, Forbes GJ, Giraldeau L-A (2006) Inadvertent social information in breeding site selection of natal dispersing birds. Proc R Soc B 273:349-355

Nurmi T, Parvinen K, Selonen V (2017) The evolution of site-selection strategy during dispersal. J Theor Biol 425:11-22. https://doi.org/ 10.1016/j.jtbi.2017.05.002

Oro D (1995) The influence of commercial fisheries in daily activity of Audouin's Gull in the Ebro Delta, NE Spain. Orn Fenn $72: 154-158$
Oro D (1998) Audouin's gull account. In: Ogilvie MA (ed) The birds of western paleartic. Oxford University Press, Oxford, pp 47-61

Oro D (2002) Breeding biology and population dynamics of Slenderbilled Gulls at the Ebro Delta (Northwestern Mediterranean). Waterbirds 25:67-77. https://doi.org/10.1675/1524-4695(2002) 025[0067:BBAPDO]2.0.CO;2

Oro D (2020) Perturbation, behavioural feedbacks, and population dynamics in social animals. Oxford University Press, Oxford

Oro D, Pradel R (1999) Recruitment of Audouin's gull to the Ebro Delta colony at metapopulation level in the western Mediterranean. Mar Ecol Prog Ser 180:267-273. https://doi.org/10.3354/ meps 180267

Oro D, Ruxton GD (2001) The formation and growth of seabird colonies: Audouin's gull as a case study. J Anim Ecol 70:527-535

Oro D, Genovart M, Ruiz X et al (1996) Differences in diet, population increase and breeding performance between two colonies of Audouin's Gulls Larus audouinii during breeding seasons affected by a trawling moratorium. J Avian Biol 27:245-251

Oro D, Pradel R, Lebreton J-D (1999) Food availability and nest predation influence life history traits in Audouin's Gull, Larus audouinii. Oecologia 118:438-445

Oro D, Pérez-Rodríguez A, Martínez-Vilalta A et al (2009) Interference competition in a threatened seabird community: a paradox for a successful conservation. Biol Conserv 142:1830-1835. https:// doi.org/10.1016/j.biocon.2009.03.023

Parejo D, Oro D, Danchin E (2006) Testing habitat copying in breeding habitat selection in a species adapted to variable environments. Ibis 148:146-154

Pärt T, Doligez B (2003) Gathering public information for habitat selection: prospecting birds cue on parental activity. Proc R Soc B 270:1809-1813. https://doi.org/10.1098/rspb.2003.2419

Pärt T, Arlt D, Doligez B et al (2011) Prospectors combine social and environmental information to improve habitat selection and breeding success in the subsequent year. J Anim Ecol 80:1227-1235. https://doi.org/10.1111/j.1365-2656.2011.01854.x

Payo-Payo A, Genovart M, Sanz-Aguilar A et al (2017) Colonisation in social species: the importance of breeding experience for dispersal in overcoming information barriers. Sci Rep 7:42866. https://doi. org/10.1038/srep42866

Payo-Payo A, Sanz-Aguilar A, Genovart M et al (2018) Predator arrival elicits differential dispersal, change in age structure and reproductive performance in a prey population. Sci Rep 8:1971. https://doi. org/10.1038/s41598-018-20333-0

Péron C, Grémillet D (2013) Tracking through life stages: adult, immature and juvenile autumn migration in a long-lived seabird. PLoS ONE 8:e72713. https://doi.org/10.1371/journal.pone.0072713

Péron G, Lebreton J-D, Crochet P-A (2010) Breeding dispersal in black-headed gull: the value of familiarity in a contrasted environment. J Anim Ecol 79:317-326. https://doi.org/10.1111/j. 1365-2656.2009.01635.x

Ponchon A, Grémillet D, Doligez B et al (2013) Tracking prospecting movements involved in breeding habitat selection: insights, pitfalls and perspectives. Methods Ecol Evol 4:143-150. https:// doi.org/10.1111/j.2041-210x.2012.00259.x

Ponchon A, Chambert T, Lobato E et al (2015a) Breeding failure induces large scale prospecting movements in the black-legged kittiwake. J Exp Mar Biol Ecol 473:138-145. https://doi.org/10. 1016/j.jembe.2015.08.013

Ponchon A, Garnier R, Grémillet D, Boulinier T (2015b) Predicting population responses to environmental change: the importance of considering informed dispersal strategies in spatially structured population models. Divers Distrib 21:88-100. https://doi.org/10. 1111/ddi.12273

Ponchon A, Iliszko L, Grémillet D et al (2017) Intense prospecting movements of failed breeders nesting in an unsuccessful breeding 
subcolony. Anim Behav 124:183-191. https://doi.org/10.1016/j. anbehav.2016.12.017

Reed JM, Boulinier T, Danchin E, Oring LW (1999) Informed dispersal. In: Nolan VJ, Ketterson ED, Thompson CF (eds) Current ornithology. Springer, Boston, pp 189-259

Rémy A, Galliard J-FL, Gundersen G et al (2011) Effects of individual condition and habitat quality on natal dispersal behaviour in a small rodent. J Anim Ecol 80:929-937. https://doi.org/10.1111/j. 1365-2656.2011.01849.x

Robinson BW, Johnson AS, Lovette IJ, Romano MD (2019) Potential northward expansion of the breeding range of Red-legged Kittiwake Rissa brevirostris. Mar Ornithol 47:229-234

Ronce O (2007) How does it feel to be like a rolling stone? Ten questions about dispersal evolution. Annu Rev Ecol Evol Syst 38:231253. https://doi.org/10.1146/annurev.ecolsys.38.091206.095611

Schippers P, Snep RPH, Schotman AGM et al (2009) Seabird metapopulations: searching for alternative breeding habitats. Popul Ecol 51:459-470. https://doi.org/10.1007/s10144-009-0159-z

Schjørring S (2002) The evolution of informed natal dispersal: inherent versus acquired information. Evol Ecol Res 4:227-238

Schmidt KA (2017) Information thresholds, habitat loss and population persistence in breeding birds. Oikos 126:651-659. https://doi.org/ 10.1111/oik.03703

Schmidt KA, Dall SRX, Van Gils JA (2010) The ecology of information: an overview on the ecological significance of making informed decisions. Oikos 119:304-316. https://doi.org/10.1111/j. 1600-0706.2009.17573.x

Schuett W, Laaksonen J, Laaksonen T (2012) Prospecting at conspecific nests and exploration in a novel environment are associated with reproductive success in the jackdaw. Behav Ecol Sociobiol 66:1341-1350. https://doi.org/10.1007/s00265-012-1389-1

Selonen V, Hanski IK (2010) Decision making in dispersing Siberian flying squirrels. Behav Ecol 21:219-225. https://doi.org/10.1093/ beheco/arp179

Serrano D, Tella JL (2003) Dispersal within a spatially structured population of lesser kestrels: the role of spatial isolation and conspecific attraction. J Anim Ecol 72:400-410
Sherer DL (2019) Variation in prospecting behavior and drivers of post-fire habitat preference among juvenile Florida scrub-jays. Master of Science, University of Central Florida

Spendelow JA, Eichenwald AJ (2018) Rapid departure of Roseate Terns (Sterna dougallii) following large-scale nest failure. Wilson J Ornithol 130:485-492. https://doi.org/10.1676/17-017.1

Stamps JA (1988) Conspecific attraction and aggregation in territorial species. Am Nat 131:329-347

Strandburg-Peshkin A, Papageorgiou D, Crofoot MC, Farine DR (2018) Inferring influence and leadership in moving animal groups. Philos Trans R Soc Lond B 373:20170006. https://doi. org/10.1098/rstb.2017.0006

Tolvanen J, Morosinotto C, Forsman JT, Thomson RL (2020) Information collected during the post-breeding season guides future breeding decisions in a migratory bird. Oecologia 192:965-977. https://doi.org/10.1007/s00442-020-04629-5

Votier SC, Grecian WJ, Patrick S, Newton J (2011) Inter-colony movements, at-sea behaviour and foraging in an immature seabird: results from GPS-PPT tracking, radio-tracking and stable isotope analysis. Mar Biol 158:355-362. https://doi.org/10.1007/ s00227-010-1563-9

Ward M (2005) Habitat selection by dispersing yellow-headed blackbirds: evidence of prospecting and the use of public information. Oecologia 145:650-657. https://doi.org/10.1007/ s00442-005-0179-0

Wilson RP, Grémillet D, Syder J et al (2002) Remote-sensing systems and seabirds: their use, abuse and potential for measuring marine environmental variables. Mar Ecol Prog Ser 228:241-261

Wolfson DW, Fieberg JR, Andersen DE (2020) Juvenile Sandhill Cranes exhibit wider ranging and more exploratory movements than adults during the breeding season. Ibis 162:556-562. https:// doi.org/10.1111/ibi.12786 